group developed only one abscess, on average, compared with the controls, which developed an average of four (J. Exp Med. 207, 1863-1870, 2010). In the future, he hopes to incorporate these proteins into conventional vaccines to more effectively protect people from acquiring $S$. aureus infections.

\section{Layers of complexity}

To banish biofilms, some scientists say you have to develop a truly biofilm-specific strategy rather than just go after $S$. aureus in any form. This philosophy gained ground in the late 1990s, when Gerald Pier, a microbiologist at Brigham and Women's Hospital in Boston, suggested an $S$. aureus vaccine directed against the sugar poly- $N$-acetyl glucosamine (PNAG), which coats the surface of bacterial biofilms. This sticky substance is an especially good choice because it is integral to the pathogen's immune evasion strategies and therefore can potentially be used to protect against all $S$. aureus infections.

In 1999, the Brigham and Women's team showed that the vaccine protected mice from a systemic $S$. aureus infection. And preinjection of PNAG-specific antibodies completely protected mice from a lethal bacterial challenge (Science 284, 1523-1527, 1999). But even though Pier took biofilms "ltann into consideration when hell out of has yet to be shown to clear biofilm infections. The that you couldn't problem, according to some cure a biofilm infection." scientists, is that PNAG is not produced by every strain of $S$. aureus, and therefore a vaccine against it might not necessarily work against all types of these stubborn infections.
Now, Shirtliff hopes to one-up Pier's PNAG-targeting vaccine by introducing a new approach directed toward S. aureus residing in the slime. His team has been hunting for key biofilm antigens, growing biofilms on medical tubing in the lab and then doing microarray analysis to tease apart which proteins are most active in the formation process on the basis of genetic signatures. Ultimately, they identified 22 cell surface proteins produced by biofilmbuilding microbes (Infect. Immun. 74, 3415$3426,2006)$. The researchers zeroed in on four proteins that were highly upregulated throughout the infection cycle and that together covered the entire biofilm. One of these, called glucosaminidase, is speculated to help break down cells surrounding the biofilm matrix to provide the bacteria there with chemical building blocks for growth.

This past April, his team reported that $87 \%$ of antibiotic-treated rabbits that previously

\title{
Persistence may pay off for antibiotics innovators
}

Among people with cystic fibrosis, nearly 90\% experience growth of biofilms that build up in their airways and clog their lungs. Existing therapies can help affected individuals breathe easier but stop short of clearing the infections. Now, scientists think they understand why: the microbes behind these infections develop a characteristic known as 'persistence'. Unlike 'resistant' microbes that can grow in the presence of antibiotics, so-called 'persistent' microbes can only tolerate these antibacterial medicines when they are dormant, which often occurs when they are in biofilms. Some experts speculate that persistent bacteria are able to escape antibiotics by turning down their internal metabolic activity.

Reporting last December, scientists at Northeastern University in Boston discovered that airway infections in people with cystic fibrosis probably recur because the microbes in the lungs have mutations that allow them to evolve persistence, which sharply decreases the effectiveness of typically prescribed antibiotics such as ofloxacin, carbenicillin and tobramycin (J. Bacteriol. 192, 6191-6199, 2010). Part of the difficulty, according to lead author Kim Lewis of Northeastern University, is that these pathogens use many mechanisms to develop persistence, making it that much harder to come up with new drugs to eradicate them.

"This Hydra has many heads," says Lewis. "Traditional, straightforward target discovery is not going to work."

The Northeastern team is now looking toward alternative strategies to develop biofilm-busting medicines. The researchers hope to develop so-called 'prodrug' antibiotics that kill pathogens only upon internalization. This is an especially good choice for persistent pathogens because the drug needs only to permeate into these microbes - the medicines eliminate these pathogens by membrane or genome disruption. Such strategies already show great promise to treat persistent tuberculosis infections.

Meanwhile, a group of researchers at Boston University School of Medicine believe that adding sugar to antibiotics might nudge

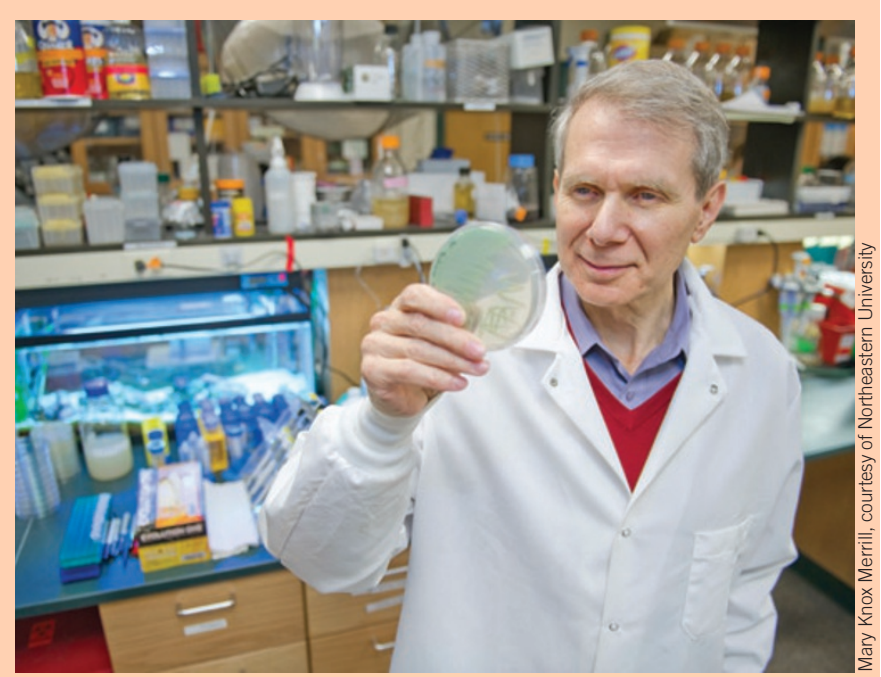

Not giving up: Kim Lewis wants to thwart persister cells.

persistent bacteria into a metabolically more active state and make them more susceptible to defeat by antibiotics. Reporting last month, James Collins and his team found that sugars made labgrown Escherichia coli and Staphylococcus aureus biofilms more vulnerable to a certain class of antibiotics called aminoglycosides. And sugar stimulated over a 90\% drop of bacterial load in antibiotic-treated mice suffering from catheter-associated infections (Nature 473, 216-220, 2011). In the future, Collins hopes to optimize his persister-busting regimen to clear these infections.

"We would like to expand the microbial arsenal," says Collins, "and make the tools we already have more effective." 